\title{
Preconditioning methods for solving a general split feasibility problem
}

Peiyuan Wang ${ }^{1,2^{*}}$, Haiyun Zhou ${ }^{2,3}$ and Yu Zhou ${ }^{2}$

Dedicated to Professor Shih-sen Chang on the occasion of his 80th birthday.

\section{${ }^{*}$ Correspondence:}

wangpeiyuan629@163.com

'The Second Training Base, Naval

Aviation Institution, Huludao,

125001, China

2Department of Mathematics, Shijiazhuang Mechanical

Engineering College, Shijiazhuang, 050003, China

Full list of author information is

available at the end of the article

\begin{abstract}
We introduce and study a new general split feasibility problem (GSFP) in a Hilbert space. This problem generalizes the split feasibility problem (SFP). The GSFP extends the SFP with a nonlinear continuous operator. We apply the preconditioning methods to increase the efficiency of the CQ algorithm, two general preconditioning $\mathrm{CQ}$ algorithms for solving the GSFP are presented. We also propose a new inexact method to approximate the preconditioner. The convergence theorems are established under the projections with respect to special norms. Some numerical results illustrate the efficiency of the proposed methods.

MSC: 46E20; 47J20; 47J25; 47L50
\end{abstract}

Keywords: general split feasibility problem; general variational inequality; preconditioning method; projection method

\section{Introduction}

As preconditioning methods can improve the condition number of the ill-posed system matrix, the convergence rate of the iterative algorithm can also be improved [1]. In [2, 3], a preconditioning method is applied to modify the projected Landweber algorithm for solving a linear feasibility problem (LFP). The modified algorithm is

$$
x_{n+1}=P_{C}\left[x_{n}-\tau D A^{*}\left(A x_{n}-b\right)\right], \quad n \geq 0,
$$

where $\tau \in\left(0,2 /\left\|D A^{*} A\right\|\right), A: X \rightarrow Y$ is a linear and continuous operator, $\|\cdot\|$ means 2-norm, $X$ and $Y$ are Hilbert spaces and $b \in Y$ is the datum of the problem, corrupted by noise or experimental errors.

While under the nonlinear conditions, Auslender and Dafermos $[4,5]$ proposed an algorithm to solve variational inequalities (VI),

$$
x_{n+1}=P_{S}\left[x_{n}-\tau_{n} G^{-1} F\left(x_{n}\right)\right], \quad n \geq 0,
$$

where $P_{S}$ is the projection operator onto $S$ with respect to the norm $\|\cdot\|_{G}$. Bertsekas and Gafni [6] and Marcotte and Wu [7] improved it with variable symmetric positive defined matrices $G_{n}$, Fukushima [8] modified it by a relaxed projection method with half-space;

\section{照 Springer}

(c)2014 Wang et al.; licensee Springer. This is an Open Access article distributed under the terms of the Creative Commons Attribution License (http://creativecommons.org/licenses/by/2.0), which permits unrestricted use, distribution, and reproduction in any medium, provided the original work is properly cited. 
then in [9], Yang established the convergence of Auslender's algorithm under the weak co-coercivity of $F$.

Further, the general variational inequality problem (GVIP) has been investigated by many authors (see [10-13]). It is to find $u^{*} \in \mathbb{R}^{n}$ such that $g\left(u^{*}\right) \in K$ and

$$
\left\langle F\left(u^{*}\right), g(u)-g\left(u^{*}\right)\right\rangle \geq 0, \quad g(u) \in K,
$$

where $K$ is a nonempty closed convex set in $\mathbb{R}^{n}, F, g: R^{n} \rightarrow R^{n}$ are nonlinear operators. In [12], Santos and Scheimberg extended and applied (1.1) to solve the GVI.

However, a general split feasibility problem (GSFP) equals to a GVI, and preconditioning methods for solving the GSFP have not been studied. By introducing a convex minimization problem, the split feasibility problem (SFP) is equivalent to a variational inequality problem (VIP), which involves a Lipschitz continuous and inversely strong monotone (ism) operator, see [14-22]. Similarly, by the same way, in this paper we introduce that a new GSFP equals to a GVI involving a Lipschitz continuous and co-coercive operator [9, 17-19].

Otherwise, Mohammad and Abdul [23] considered a general split feasibility in infinitedimensional real Hilbert spaces. It is to find $x^{*}$ such that

$$
x^{*} \in \bigcap_{i=1}^{\infty} C_{i}, \quad A x^{*} \in \bigcap_{j=1}^{\infty} Q_{j},
$$

where $A: H_{1} \rightarrow H_{2}$ is a bounded linear operator, $\left\{C_{i}\right\}_{i=1}^{\infty}$ and $\left\{Q_{j}\right\}_{j=1}^{\infty}$ are the families of nonempty closed convex subsets of $H_{1}$ and $H_{2}$, respectively.

Let $C$ and $Q$ be nonempty closed convex subsets in real Hilbert spaces $H_{1}$ and $H_{2}$, respectively. We consider a general split feasibility problem which is different from the one in [23]. Our GSFP is to find

$$
x^{*} \in H_{1}, g\left(x^{*}\right) \in C \text { such that } A g\left(x^{*}\right) \in Q \text {, }
$$

where $A: H_{1} \rightarrow H_{2}$ is a bounded linear operator and $g: H_{1} \rightarrow C$ is a continuous operator. We see that the SFP in [24] and the GSFP in [23] are particular cases of GSFP (1.2). It has applications in many special fields such as signal decryption, demodulating the digital signal and noise processing, etc. In order to solve GSFP (1.2), two preconditioning algorithms are developed in this paper following the iterative scheme

$$
g\left(x_{n+1}\right)=P_{C}\left[g\left(x_{n}\right)-\gamma D A^{*}\left(I-P_{Q}\right) A g\left(x_{n}\right)\right], \quad n \geq 0,
$$

where the two general constraints $C$ and $Q$ deal with projections with respect to the norms corresponding to some symmetric positive definite matrices. Define the solution set of (1.2) $\Gamma=\left\{x^{*} \in H_{1} \mid A g\left(x^{*}\right) \in Q\right\}$, as $\Gamma$ is nonempty, and by virtue of the related G-cocoercive operator, we can establish the convergence of the proposed algorithms.

The paper is organized as follows. Section 2 presents two useful propositions. In Section 3, we define the algorithms with fixed preconditioner, variable preconditioner, relaxed projection and preconditioner approximation and analyze the convergence. Numerical results are reported in Section 4. Finally, Section 5 gives some concluding remarks. 


\section{Preliminaries}

In what follows, we state some concepts and propositions.

The SFP is to find a point $x^{*} \in C$ such that $A x^{*} \in Q$, where $A: H_{1} \rightarrow H_{2}$ is a bounded linear operator [24].

Let $G$ be a symmetric positive definite matrix, and set $D=G^{-1}$. Then the norm $\|\cdot\|_{G}$ is defined by $\|x\|_{G}^{2}=\langle x, G x\rangle$ for $\forall x \in H$. We denote by $P_{C}$ the projection operator onto $C$ with respect to the norm $\|\cdot\|_{G}[6]$, i.e.,

$$
P_{C}(x)=\arg \min _{y \in C}\left\{\|x-y\|_{G}\right\} .
$$

Let $\lambda_{\min }$ and $\lambda_{\max }$ be the minimum and the largest eigenvalues of $G$, respectively. Then for the 2-norm $\|\cdot\|[18,19]$, we have

$$
\lambda_{\min }\|x\|^{2} \leq\|x\|_{G}^{2} \leq \lambda_{\max }\|x\|^{2}, \quad \forall x \in H
$$

Proposition 2.1 $[7,10]$ Let $C$ be a nonempty closed convex subset in $H$, for $\forall x, y \in H$ and $\forall z \in C$, the G-projection operator onto $C$ has the following properties:

(i) $\left\langle G\left(x-P_{C} x\right), z-P_{C}(x)\right\rangle \leq 0$;

(ii) $\|x \pm y\|_{G}^{2}=\|x\|_{G}^{2} \pm 2\langle x, G y\rangle+\|y\|_{G}^{2}$;

(iii) $\left\|P_{C}(x)-P_{C}(y)\right\|_{G}^{2} \leq\|x-y\|_{G}^{2}-\left\|\left(P_{C}(x)-x\right)-\left(P_{C}(y)-y\right)\right\|_{G}^{2}$.

Let $\tilde{D}$ be a symmetric positive definite matrix, and $A^{T} \tilde{D}=D A^{T}$. Then the norm $\|\cdot\|_{\tilde{D}}$ is defined by $\|y\|_{\tilde{D}}^{2}=\langle y, \tilde{D} y\rangle$ for $\forall y \in H$. We denote by $P_{Q}$ the projection operator onto $Q$ with respect to the norm $\|\cdot\|_{\tilde{D}}$. According to the SFP, when GSFP (1.2) has no solution (refer to $[2,14,15])$, we can define

$$
f_{g}(x)=\frac{1}{2}\left\|\left(I-P_{Q}\right) A g(x)\right\|^{2}
$$

and

$$
f_{\tilde{D}}^{g}(x)=\frac{1}{2}\left\|A g(x)-P_{Q} A g(x)\right\|_{\tilde{D}}^{2}=\left\langle\tilde{D}\left(A g(x)-P_{Q} A g(x)\right), A g(x)-P_{Q} A g(x)\right\rangle,
$$

$f_{\tilde{D}}^{g}(x)$ is also convex and continuously differentiable in $H$. Its gradient operator is

$$
\nabla f_{D}^{g}(x)=D A^{T}\left(I-P_{Q}\right) A g(x)
$$

As $D=I$, we define

$$
\nabla f_{g}(x)=A^{T}\left(I-P_{Q}\right) A g(x)
$$

$\nabla f_{g}(x)$ is also Lipschitz continuous.

Proposition 2.2 If we consider the constrained minimization problem

$$
\min \left\{f_{\tilde{D}}^{g}(x) \mid x \in H_{1} \text { s.t. } g(x) \in C\right\}
$$


its stationary point $x^{*} \in H_{1}$ satisfies

$$
\left\{\begin{array}{l}
x^{*} \in H, g\left(x^{*}\right) \in C \text { such that } \\
\left\langle\nabla f_{D}^{g}\left(x^{*}\right), g(x)-g\left(x^{*}\right)\right\rangle \geq 0, \quad \forall g(x) \in C,
\end{array}\right.
$$

which is a general variational inequality involving a Lipschitz continuous and G-cocoercive operator.

Proof For $\forall x, y \in H$, from (2.1) and Lemma 8.1 in [15], we have

$$
\begin{aligned}
\left\|\nabla f_{D}^{g}(x)-\nabla f_{D}^{g}(y)\right\|_{G}^{2} & \leq \lambda_{\max }(G)\left\|\nabla f_{D}^{g}(x)-\nabla f_{D}^{g}(y)\right\|^{2} \\
& \leq \frac{\lambda_{\max }^{2}(D)}{\lambda_{\min }(D)}\left\|\nabla f_{g}(x)-\nabla f_{g}(y)\right\|^{2} \\
& \leq \frac{\lambda_{\max }^{2}(D)}{\lambda_{\min }(D)} L^{2}\|g(x)-g(y)\|^{2} \\
& \leq \frac{\lambda_{\max }^{3}(D)}{\lambda_{\min }(D)} L^{2}\|g(x)-g(y)\|_{G}^{2}
\end{aligned}
$$

where $L$ is the largest eigenvalue of $A^{T} A$; therefore, the operator $\nabla f_{D}^{g}$ is Lipschitz continuous,

$$
\begin{aligned}
& \left\langle\nabla f_{D}^{g}(x)-\nabla f_{D}^{g}(y), g(x)-g(y)\right\rangle \\
& \quad \geq \lambda_{\min }(D)\left\langle\nabla f_{g}(x)-\nabla f_{g}(y), g(x)-g(y)\right\rangle \\
& \quad \geq \frac{\lambda_{\min }(D)}{L}\left\|\nabla f_{g}(x)-\nabla f_{g}(y)\right\|^{2} \\
& \quad \geq \frac{\lambda_{\min }(D)}{L \cdot \lambda_{\max }(D)}\left\|\nabla f_{g}(x)-\nabla f_{g}(y)\right\|_{D}^{2} \\
& \quad=\frac{\lambda_{\min }(D)}{L \cdot \lambda_{\max }(D)}\left\langle D\left(\nabla f_{g}(x)-\nabla f_{g}(y)\right), G D\left(\nabla f_{g}(x)-\nabla f_{g}(y)\right)\right\rangle \\
& \quad=\frac{\lambda_{\min }(D)}{L \cdot \lambda_{\max }(D)}\left\|\nabla f_{D}^{g}(x)-\nabla f_{D}^{g}(y)\right\|_{G}^{2} .
\end{aligned}
$$

Thus, the operator $\nabla f_{D}^{g}$ is co-coercive.

\section{Main results}

In this section, we propose several modified CQ algorithms with preconditioning techniques and prove the convergence.

\subsection{General preconditioning CQ algorithm}

In this part, we have our first algorithm with fixed stepsize and preconditioner to solve GSFP (1.2). The algorithm is as follows.

Algorithm 3.1 Choose $\forall x_{0} \in H_{1}$ such that $g\left(x_{0}\right) \in C$, and let $x_{n} \in H_{1}$ such that $g\left(x_{n}\right) \in C$, then we calculate $x_{n+1}$ such that

$$
g\left(x_{n+1}\right)=P_{C}\left[g\left(x_{n}\right)-\gamma \nabla f_{D}^{g}\left(x_{n}\right)\right], \quad n \geq 0,
$$

where $\gamma \in\left(0, \frac{2}{L \cdot L_{D}}\right), L$ and $L_{D}$ are the largest eigenvalues of $A^{T} A$ and $D$, respectively. 
Now we establish the weak convergence of Algorithm 3.1.

Theorem 3.1 Suppose that the operators $g: H_{1} \rightarrow C$ and $g^{-1}: C \rightarrow H_{1}$ are continuous. If $\Gamma \neq \emptyset$, then the sequence $\left\{x_{n}\right\}$ generated by Algorithm 3.1 converges to the solution of GSFP (1.2).

Proof Firstly, for $\forall x^{*} \in \Gamma$, we have

$$
g\left(x^{*}\right)=P_{C}\left[g\left(x^{*}\right)-\gamma \nabla f_{D}^{g}\left(x^{*}\right)\right] .
$$

From (3.1), (iii), (ii) and the definition of ism, we have

$$
\begin{aligned}
\| & g\left(x_{n+1}\right)-g\left(x^{*}\right) \|_{G}^{2} \\
= & \left\|P_{C}\left[g\left(x_{n}\right)-\gamma \nabla f_{D}^{g}\left(x_{n}\right)\right]-P_{C}\left[g\left(x^{*}\right)-\gamma \nabla f_{D}^{g}\left(x^{*}\right)\right]\right\|_{G}^{2} \\
\leq & \left\|g\left(x_{n}\right)-g\left(x^{*}\right)-\gamma\left[\nabla f_{D}^{g}\left(x_{n}\right)-\nabla f_{D}^{g}\left(x^{*}\right)\right]\right\|_{G}^{2} \\
\leq & \left\|g\left(x_{n}\right)-g\left(x^{*}\right)\right\|_{G}^{2}-2 \gamma\left(g\left(x_{n}\right)-g\left(x^{*}\right), \nabla f_{g}\left(x_{n}\right)-\nabla f_{g}\left(x^{*}\right)\right\rangle \\
& \quad+\gamma^{2}\left\langle\nabla f_{D}^{g}\left(x_{n}\right)-\nabla f_{D}^{g}\left(x^{*}\right), \nabla f_{g}\left(x_{n}\right)-\nabla f_{g}\left(x^{*}\right)\right\rangle \\
\leq & \left\|g\left(x_{n}\right)-g\left(x^{*}\right)\right\|_{G}^{2}-\left(\frac{2 \gamma}{L}-\gamma^{2} L_{D}\right)\left\|\nabla f_{g}\left(x_{n}\right)-\nabla f_{g}\left(x^{*}\right)\right\|^{2},
\end{aligned}
$$

as $\frac{2 \gamma}{L}-\gamma^{2} L_{D}>0$, which implies that the sequence $\left\{\left\|g\left(x_{n}\right)-g\left(x^{*}\right)\right\|_{G}\right\}_{n \in \mathbb{N}}$ is monotonically decreasing, then we can obtain that the sequence $\left\{\left\|g\left(x_{n}\right)-g\left(x^{*}\right)\right\|_{G}\right\}_{n \in \mathbb{N}}$ is also convergent, especially, the sequence $\left\{g\left(x_{n}\right)\right\}_{n \in \mathbb{N}}$ is bounded. Consequently, we get from (3.2)

$$
\lim _{n \rightarrow \infty}\left\|\nabla f_{g}\left(x_{n}\right)-\nabla f_{g}\left(x^{*}\right)\right\|=\lim _{n \rightarrow \infty}\left\|\nabla f_{g}\left(x_{n}\right)\right\|=0
$$

Moreover, for each $g\left(x_{n}\right) \in C$, from (iii) and (2.1), we have

$$
\begin{aligned}
\left\|g\left(x_{n+1}\right)-g\left(x_{n}\right)\right\|_{G} & \leq \gamma\left\|\nabla f_{D}\left(x_{n}\right)\right\|_{G} \\
& \leq \gamma L_{D}\left\|\nabla f_{g}\left(x_{n}\right)\right\|_{G} \leq \gamma L_{D} \sqrt{\lambda_{\max }(G)}\left\|\nabla f_{g}\left(x_{n}\right)\right\| .
\end{aligned}
$$

Then by virtue of (3.3) we have

$$
\lim _{n \rightarrow \infty}\left\|g\left(x_{n+1}\right)-g\left(x_{n}\right)\right\|_{G}=0 .
$$

Hence, there exists a subsequence $\left\{g\left(x_{j}\right)\right\}_{j \in \bar{N}}$ of $\left\{g\left(x_{n}\right)\right\}_{n \in \mathbb{N}}$ such that

$$
\lim _{j \rightarrow \infty}\left\|g\left(x_{j+1}\right)-g\left(x_{j}\right)\right\|_{G}=0 .
$$

Thus, $\left\{g\left(x_{j}\right)\right\}_{j \in \bar{N}}$ is also bounded.

Let $\bar{x}$ be an accumulation point of $\left\{x_{n}\right\}$, then the subsequence of $\left\{x_{n}\right\},\left\{x_{j}\right\}_{j \in \bar{N}} \rightarrow \bar{x}$ as $j \rightarrow \infty$. Because of the continuity of $g$, there exists an accumulation point $g(\bar{x}) \in C$ of the 
sequence $\left\{g\left(x_{n}\right)\right\}_{n \in \mathbb{N}}$; for the subsequence $\left\{g\left(x_{j}\right)\right\}_{j \in \bar{N}}$, we have $\left\{g\left(x_{j}\right)\right\}_{j \in \bar{N}} \rightarrow g(\bar{x})$ as $j \rightarrow \infty$. After that, from (3.3) we obtain

$$
\lim _{j \rightarrow \infty}\left\|\nabla f_{g}\left(x_{n_{j}}\right)\right\|=\left\|\nabla f_{g}(\bar{x})\right\|=\left\|A^{T} A g(\bar{x})-A^{T} P_{Q} A g(\bar{x})\right\|=0,
$$

that is, $\operatorname{Ag}(\bar{x}) \in Q$.

We use $\bar{x}$ in place of $x^{*}$ in (3.2) and obtain that $\left\{\left\|g\left(x_{n}\right)-g(\bar{x})\right\|_{G}\right\}$ is convergent. Because its subsequence $\left\{\left\|g\left(x_{n_{j}}\right)-g(\bar{x})\right\|_{G}\right\} \rightarrow 0$, then we get that $\left\{g\left(x_{n}\right)\right\}_{n \in \mathbb{N}}$ converges to $g(\bar{x})$ as $j \rightarrow \infty$. As well as $g^{-1}$ is continuous, we finally have

$$
\lim _{n \rightarrow \infty} x_{n}=\bar{x} \in \Gamma
$$

Therefore, $\bar{x}$ is a solution of GSFP (1.2).

From Algorithm 3.1 and Theorem 3.1 we can deduce the following results easily.

Corollary 3.1 If $g=I$, then GSFP (1.2) reduces to SFP, Algorithm 3.1 also reduces to a preconditioning CQ (PCQ) algorithm for $\forall x_{0} \in H_{1}$ :

$$
x_{n+1}=P_{C}\left[x_{n}-\gamma \nabla f_{D}\left(x_{n}\right)\right], \quad n \geq 0,
$$

where $\gamma \in\left(0, \frac{2}{L \cdot L_{D}}\right)$, L and $L_{D}$ are the largest eigenvalues of $A^{T} A$ and $D$, respectively. $P_{C}$ and $P_{Q}$ are still the projection operators onto $C$ and $Q$ with respect to the norm $\|\cdot\|_{D}$ and $\|\cdot\|_{\tilde{D}}$, respectively.

Corollary 3.2 If $g=I, D=I, \tilde{D}=I$, then GSFP reduces to SFP, then Algorithm 3.1 reduces to the CQ algorithm proposed in [25].

Corollary 3.3 If $g=I, \tilde{D}=I, P_{C}$ and $P_{Q}$ are the projections onto $C$ and $Q$ with respect to the norm $\|\cdot\|$, set $F\left(x_{n}\right)=D A^{T}\left(I-P_{Q}\right) A D x_{n}$, (3.5) transforms into the algorithm in [26]

$$
x_{n+1}=P_{C}\left[x_{n}-\gamma F\left(x_{n}\right)\right], \quad n \geq 0,
$$

where $\gamma \in\left(0, \frac{2}{L}\right), L=\left\|D A^{T}\right\|^{2}$. Then the GSFP reduces to the extended split feasibility problem $($ ESFP) in [26].

\subsection{An algorithm with variable projection metric}

The algorithms above can speed the convergence of CQ algorithm, but the stepsize and preconditioner are fixed. In this subsection, we extend the results in [6] and construct an iterative scheme with variable stepsize and preconditioner $D_{n}$ from one iteration to the next. As a key role, $D_{n}$ will change arbitrarily or following some rules to achieve the convergence progress and better results.

Let $D_{n}$ and $\tilde{D}_{n}$ be two symmetric positive definite matrices for $n=0,1,2, \ldots$ Denote by $P_{C}$ and $P_{Q}$ the projections onto $C$ and $Q$ with respect to the norm $\|\cdot\|_{D_{n}}$ and $\|\cdot\|_{\tilde{D}_{n}}$, respectively. Let $\chi$ be a set of symmetric positive definite matrices, we have the following algorithm. 
Algorithm 3.2 Choose $\forall x_{0} \in H_{1}$ such that $g\left(x_{0}\right) \in C$, and let $x_{n} \in H_{1}$ such that $g\left(x_{n}\right) \in C$; for $\forall D_{n} \in \chi$, we compute $x_{n+1}$ such that

$$
g\left(x_{n+1}\right)=P_{C}\left[g\left(x_{n}\right)-\gamma_{n} D_{n} \nabla f_{g}\left(x_{n}\right)\right], \quad n \geq 0,
$$

where $\gamma_{n} \in\left(0, \frac{2}{L \cdot M_{D}}\right), L$ is the largest eigenvalue of $A^{T} A, M_{D}$ is the minimum value of all the largest eigenvalue values $L_{D_{n}}$ to matrices $D_{n}$.

Remark 3.1 Define $d_{n}=\left\|g\left(x_{n+1}\right)-g\left(x_{n}\right)\right\|_{G_{n}}$, then for the next iteration, $D_{n+1}$ is either chosen arbitrarily from $\chi$ or equivalent to $D_{n}$. It is conditional on whether $d_{n}$ has decreased or not. More particularly, we define a scalar $\bar{d}_{n}$ with initial value $\bar{d}_{0}=\infty$. Having chosen a scalar $\alpha \in(0,1)$ at the $n$th iteration, then $\bar{d}_{n+1}$ is calculated by

$$
\bar{d}_{n+1}= \begin{cases}\alpha \bar{d}_{n}, & \text { if } d_{n} \leq \bar{d}_{n} ; \\ \bar{d}_{n}, & \text { if } d_{n}>\bar{d}_{n},\end{cases}
$$

then we select

$$
D_{n+1}= \begin{cases}\forall D \in \chi, & \text { if } \bar{d}_{n+1}<\bar{d}_{n} ; \\ D_{n}, & \text { if } \bar{d}_{n+1}=\bar{d}_{n} .\end{cases}
$$

Theorem 3.2 If $\Gamma \neq \emptyset$, then the sequence $\left\{x_{n}\right\}$ generated by Algorithm 3.2 converges to the solution of GSFP (1.2).

Proof To obtain variable $D_{n}$ at each iteration and keep the convergence of Algorithm 3.2, $d_{n}$ must be a descending behavior for $n=0,1,2, \ldots$. We first show that

$$
\varliminf_{n \rightarrow \infty} d_{n}=0 .
$$

Indeed if (3.7) is not true, we have $\underline{\lim }_{n \rightarrow \infty} d_{n}>0$, then $D_{n}$ must have changed a finite number of times, we set that this number is $\kappa \in N$. Therefore, let $x^{*} \in \Gamma$ be a solution of GSFP, refer to (3.2) and for $n>\kappa$, we have

$$
\begin{aligned}
\left\|g\left(x_{n+1}\right)-g\left(x^{*}\right)\right\|_{G_{\kappa}}^{2} \leq & \left\|g\left(x_{n}\right)-g\left(x^{*}\right)\right\|_{G_{\kappa}}^{2} \\
& -\left(\frac{2 \gamma_{\kappa}}{L}-\gamma_{\kappa}^{2} L_{D_{\kappa}}\right)\left\|\nabla f_{g}\left(x_{n}\right)-\nabla f_{g}\left(x^{*}\right)\right\|^{2},
\end{aligned}
$$

as $M_{D}=\min \left\{L_{D_{n}} \mid n=0,1, \ldots \kappa\right\}, \frac{2 \gamma_{\kappa}}{L}-\gamma_{\kappa}^{2} L_{D_{\kappa}}>0$. Then, following the proof of Theorem 3.1, we also get

$$
\lim _{n \rightarrow \infty}\left\|\nabla f_{g}\left(x_{n}\right)\right\|=0
$$

and

$$
\lim _{n \rightarrow \infty} d_{n}=\lim _{n \rightarrow \infty}\left\|g\left(x_{n+1}\right)-g\left(x_{n}\right)\right\|_{G_{\kappa}}=0 .
$$

Equation (3.9) contradicts the above hypothesis, so (3.7) is true. 
By using (3.6) and (iii), for the $n$th iteration, we have

$$
\begin{aligned}
d_{n} & =\left\|g\left(x_{n+1}\right)-g\left(x_{n}\right)\right\|_{G_{n}}^{2} \\
& \geq \frac{1}{2}\left\|g\left(x_{n}\right)-P_{C}\left[g\left(x_{n}\right)\right]\right\|_{G_{n}}^{2}-\left\|g\left(x_{n+1}\right)-P_{C}\left[g\left(x_{n}\right)\right]\right\|_{G_{n}}^{2} \\
& =\frac{1}{2}\left\|g\left(x_{n}\right)-P_{C}\left[g\left(x_{n}\right)\right]\right\|_{G_{n}}^{2}-\left\|P_{C}\left[g\left(x_{n}\right)-\gamma_{n} D_{n} \nabla f_{g}\left(x_{n}\right)\right]-P_{C}\left[g\left(x_{n}\right)\right]\right\|_{G_{n}}^{2} \\
& \geq \frac{1}{2}\left\|g\left(x_{n}\right)-P_{C}\left[g\left(x_{n}\right)\right]\right\|_{G_{n}}^{2}-\gamma_{n} L_{D_{n}}\left\|\nabla f_{g}\left(x_{n}\right)\right\|_{G_{n}}^{2},
\end{aligned}
$$

where $\gamma_{n} L_{D_{n}}>0$. Then from (2.1) and (3.8) we know that

$$
\lim _{n \rightarrow \infty}\left\|\nabla f_{g}\left(x_{n}\right)\right\|_{G_{n}}=\lim _{n \rightarrow \infty}\left\|A^{T}\left(I-P_{Q}\right) A g\left(x_{n}\right)\right\|_{G_{n}}=0
$$

so $A g\left(x_{n}\right) \in Q$. By virtue of (3.7), we also have

$$
\frac{\lim }{n}\left\|g\left(x_{n}\right)-P_{C}\left[g\left(x_{n}\right)\right]\right\|=0 .
$$

This means that at least a subsequence of $\left\{g\left(x_{n}\right)\right\}_{n \in \mathbb{N}}$ converges to a solution of $g(\bar{x}) \in \Gamma$. Similar to the argumentation of accumulation in the proof of Theorem 3.1, we know that $\left\{x_{n}\right\}$ converges to a solution of GSFP.

\subsection{Some methods for execution}

In Algorithms 3.1 and 3.2, there still exists difficulty to implement the projections $P_{C}$ and $P_{Q}$ with respect to the defined norms, especially when $C$ and $Q$ are general closed convex sets. According to the relaxed method in $[8,27,28]$, we consider the above algorithm in which the closed convex subsets $C$ and $Q$ are the following particular formula:

$$
C=\left\{g(x) \in H_{1} \mid c(g(x)) \leq 0\right\} \quad \text { and } \quad Q=\left\{A g(x) \in H_{2} \mid q(A g(x)) \leq 0\right\}
$$

where $c: H_{1} \rightarrow \mathbb{R}$ and $q: H_{2} \rightarrow \mathbb{R}$ are convex functions. $C_{n}$ and $Q_{n}$ are given as

$$
\begin{aligned}
& C_{n}=\left\{g(x) \in H_{1} \mid c\left(g\left(x_{n}\right)\right)+\left\langle\xi_{n}, g(x)-g\left(x_{n}\right)\right\rangle \leq 0\right\} \\
& Q_{n}=\left\{A g(x) \in H_{1} \mid q\left(A g\left(x_{n}\right)\right)+\left\langle\eta_{n}, A g(x)-A g\left(x_{n}\right)\right\rangle \leq 0\right\}
\end{aligned}
$$

where $\xi_{n} \in \partial c\left(g\left(x_{n}\right)\right), \eta_{n} \in \partial q\left(A g\left(x_{n}\right)\right)$.

Here, we also replace $P_{C}$ and $P_{Q}$ by $P_{C_{n}}$ and $P_{Q_{n}}$. However, in this paper, take Algorithm 3.2 for example, the projections are with respect to the norms corresponding to $G_{n}$ and $\tilde{D}_{n}$, we should use the following methods to calculate them. For $\forall z \in H_{1}$ and $\forall y \in H_{2}$,

$$
P_{C_{n}}(z)= \begin{cases}z-D_{n} \frac{c\left[g\left(x_{n}\right)\right]+\left\langle D_{n} \xi_{n}, z-g\left(x_{n}\right)\right\rangle}{\left\|\xi_{n}\right\|_{D_{n}}^{2}} \xi_{n}, & \text { if } c\left[g\left(x_{n}\right)\right]+\left\langle D_{n} \xi_{n}, z-g\left(x_{n}\right)\right\rangle>0 \\ z, & \text { otherwise }\end{cases}
$$

and

$$
P_{Q_{n}}(y)= \begin{cases}y-\tilde{D}_{n} \frac{q\left[A g\left(x_{n}\right)\right]+\left\langle\tilde{D}_{n} \eta_{n}, y-A g\left(x_{n}\right)\right\rangle}{\left\|\eta_{n}\right\|_{\tilde{D}_{n}}^{2}} \eta_{n}, & \text { if } q\left[A g\left(x_{n}\right)\right]+\left\langle\tilde{D}_{n} \eta_{n}, y-A g\left(x_{n}\right)\right\rangle>0 \\ y, & \text { otherwise. }\end{cases}
$$


Set $z=g\left(x_{n}\right)-\gamma_{n} D_{n} \nabla f_{g}\left(x_{n}\right), y=A g\left(x_{n}\right)$, let $\bar{x} \in H_{1}$ be an accumulation of $\left\{x_{n}\right\}_{n \in \mathbb{N}}$. From the proof above, it is easy to deduce that

$$
\begin{aligned}
& \lim _{n \rightarrow \infty} c\left[g\left(x_{n}\right)\right]+\left\langle D_{n} \xi_{n}, z-g\left(x_{n}\right)\right\rangle=c[g(\bar{x})] \leq 0, \\
& \lim _{n \rightarrow \infty} q\left[A g\left(x_{n}\right)\right]+\left\langle\tilde{D}_{n} \eta_{n}, y-A g\left(x_{n}\right)\right\rangle=q[A g(\bar{x})] \leq 0 .
\end{aligned}
$$

Therefore, $g(\bar{x}) \in C \subseteq C_{n}, A g(\bar{x}) \in Q \subseteq Q_{n}$, with the projections $P_{C_{n}}$ and $P_{Q_{n}}, \bar{x}$ is a solution of GSFP.

Next, we present a new approximation method to estimate $\gamma_{n}$ and $D_{n}$ in Algorithm 3.2. If $\Gamma \neq \emptyset$, for $\forall x^{*} \in \Gamma$ and $n \geq 0$, such that

$$
D_{n} \nabla f_{g}\left(x^{*}\right)=D_{n} A^{T} A g\left(x^{*}\right)-D_{n} A^{T} P_{Q_{n}} A g\left(x^{*}\right)=0,
$$

under the ideal condition, if $D_{n} A^{T} A \approx I$, the solution is done, but unfortunately, $\left(A^{T} A\right)^{-1}$ cannot be calculated directly when $A$ is a large matrix in practice. As

$$
A^{T} A g\left(x^{*}\right)=A^{T} P_{Q_{n}} A g\left(x^{*}\right)=\lambda g\left(x^{*}\right),
$$

where $\lambda$ is an eigenvalue of $A^{T} A$. Let $D_{0}=I$, for the $n$th iteration, we have the next $j \times j$ approximation of $D_{n+1}$

$$
D_{n+1}^{j j}= \begin{cases}\frac{\left[g\left(x_{n}\right)\right]_{j}}{\left[A^{T} P_{Q_{n}} A g\left(x_{n}\right)\right]_{j}}, & \text { if }\left[g\left(x_{n}\right)\right]_{j} \neq 0 \text { and }\left[A^{T} P_{Q_{n}} A g\left(x_{n}\right)\right]_{j} \neq 0 ; \\ D_{n}^{j j}, & \text { otherwise, }\end{cases}
$$

where $j=1,2, \ldots$. So, at the $n$th iteration, let $l_{D_{n}}$ be the minimum eigenvalue of $D_{n}$, take $M_{D_{n}} \approx \min \left\{L_{D_{k}} \mid k=0,1, \ldots, n\right\}, L_{n} \approx \max \left\{\left(l_{D_{k}}\right)^{-1} \mid k=0,1, \ldots, n\right\}$, the variable stepsize is approximated by

$$
\gamma_{n}=\frac{\rho_{n}}{L_{n} \cdot M_{D_{n}}}, \quad \rho_{n} \in(0,2), n=1,2, \ldots
$$

\section{Numerical results}

We consider the following problem from [29] in a finite dimensional Hilbert space:

Let $C=\left\{x \in H_{1} \mid c(x) \leq 0\right\}$, where $c(x)=-x_{1}+x_{2}^{2}+\cdots+x_{N}^{2}$, and $Q=\left\{y \in H_{2} \mid q(y) \leq 0\right\}$, where $q(y)=y_{1}+y_{2}^{2}+\cdots+y_{M}^{2}-1 . A_{M \times N}$ is a random matrix where every element of $A$ is in $(0,1)$ satisfying $\Gamma \neq \emptyset$. Let $x_{0}$ be a random vector in $H_{1}$ where every element of $x_{0}$ is in $(0,1)$.

We set $\left\|x_{n+1}-x_{n}\right\| \leq \varepsilon$ as the stop rule, and let $N=10, M=20, g=I, \tilde{D}_{n}=I$, for $n \geq 0$. Using the methods in Section 3.3, we compare Algorithm 3.2 with the relaxed CQ algorithm (RCQ) in [30], with different $\varepsilon$ and initial values. The results can be seen in Table 1 . We see that the proposed methods in this paper behave better.

\section{Concluding remarks}

In this paper, we have discussed a new general split feasibility problem, which is related to the general variational inequalities involving a co-coercive operator. By using the $G$-norm 
Table 1 The comparison between the results of preconditioning and relaxed CQ algorithms

\begin{tabular}{llrlll}
\hline $\boldsymbol{\varepsilon}$ & Algorithms & \multicolumn{1}{c}{$\boldsymbol{n}$} & CPU (s) & $\boldsymbol{c ( x )}$ & $\boldsymbol{q}(\boldsymbol{y})$ \\
\hline 0.01 & RCQ & 16 & 0.0112 & -0.1665 & 57.1092 \\
& 3.2 & 22 & 0.0049 & -0.1333 & 1.2636 \\
0.001 & RCQ & 200 & 0.0216 & -0.2684 & 1.2708 \\
& 3.2 & 13 & 0.0039 & -0.0770 & $3.5300 \mathrm{E}-02$ \\
0.0001 & RCQ & 360 & 0.0295 & -0.1039 & $1.0710 \mathrm{E}-01$ \\
& 3.2 & 60 & 0.0081 & -0.0479 & $1.4900 \mathrm{E}-02$ \\
0.00001 & RCQ & 464 & 0.0363 & -0.2561 & $7.5000 \mathrm{E}-03$ \\
& 3.2 & 21 & 0.0043 & -0.0566 & $3.3809 \mathrm{E}-04$ \\
\hline
\end{tabular}

method, variable modulus method and relaxed method, two modified projection algorithms for solving the GSFP and some approximate methods for algorithm executing have been presented. The numerical results show that by preconditioning method, the convergence speed of CQ algorithm can be improved, but the way to obtain variable stepsize in the paper is inexact. To continue to improve it or combine it with the methods in [14] and [28] is another interesting subject.

\section{Competing interests}

The authors declare that they have no competing interests.

\section{Authors' contributions}

The authors take equal roles in deriving results and writing of this paper. All authors read and approved the final manuscript.

\section{Author details}

${ }^{1}$ The Second Training Base, Naval Aviation Institution, Huludao, 125001, China. ${ }^{2}$ Department of Mathematics, Shijiazhuang Mechanical Engineering College, Shijiazhuang, 050003, China. ${ }^{3}$ Department of Mathematics and Information, Hebei Normal University, Shijiazhuang, 050024, China.

\section{Acknowledgements}

The authors would like to thank the associate editor and the referees for their comments and suggestions. This research was supported by the National Natural Science Foundation of China (11071053)

Received: 14 February 2014 Accepted: 17 October 2014 Published: 31 Oct 2014

\section{References}

1. Chen, K: Matrix Preconditioning Techniques and Applications. Cambridge University Press, New York (2005)

2. Piana, M, Bertero, M: Projected Landweber method and preconditioning. Inverse Probl. 13, 441-463 (1997)

3. Strand, ON: Theory and methods related to the singular-function expansion and Landweber's iteration for integral equations of the first kind. SIAM J. Numer. Anal. 11, 798-824 (1974)

4. Auslender, A: Optimisation: Méthodes Numérique. Masson, Paris (1976)

5. Dafermos, S: Traffic equilibrium and variational inequalities. Transp. Sci. 14, 42-54 (1980)

6. Bertsekas, PD, Gafni, EM: Projection methods for variational inequities with application to the traffic assignment problem. Math. Program. Stud. 17, 139-159(1982)

7. Marcotte, $\mathrm{P}, \mathrm{Wu}, \mathrm{JH}$ : On the convergence of projection methods: application to the decomposition of affine variational inequalities. J. Optim. Theory Appl. 85, 347-362 (1995)

8. Fukushima, M: A relaxed projection method for variational inequalities. Math. Program. 35, 58-70 (1986)

9. Yang, Q: The revisit of a projection algorithm with variable steps for variational inequalities. J. Ind. Manag. Optim. 1, 211-217 (2005)

10. He, BS: Inexact implicit methods for monotone general variational inequalities. Math. Program. 86, $199-217$ (1999)

11. Noor, MA, Wang, YJ, Xiu, N: Projection iterative schemes for general variational inequalities. J. Inequal. Pure Appl. Math. 3, Article 34 (2002)

12. Santos, PSM, Scheimberg, S: A projection algorithm for general variational inequalities with perturbed constraint sets. Appl. Math. Comput. 181, 649-661 (2006)

13. Muhammad, AN, Abdellah, B, Saleem, U: Self-adaptive methods for general variational inequalities. Nonlinear Anal. 71, 3728-3738 (2009)

14. Qu, B, Xiu, N: A note on the CQ algorithm for the split feasibility problem. Inverse Probl. 21, 1655-1665 (2005)

15. Byrne, $\mathrm{C}$ : A unified treatment of some iterative algorithms in signal processing and image reconstruction. Inverse Probl. 20, 103-120 (2004)

16. Dolidze, Z: Solution of variational inequalities associated with a class of monotone maps. Èkon. Mat. Metody 18 925-927 (1982)

17. He, B, He, X, Liu, H, Wu, T: Self-adaptive projection method for co-coercive variational inequalities. Eur. J. Oper. Res. 196, 43-48 (2009) 
18. Facchinei, F, Pang, JS: Finite-Dimensional Variational Inequality and Complementarity Problems, vol. I. Springer, New York (2003)

19. Facchinei, F, Pang, JS: Finite-Dimensional Variational Inequality and Complementarity Problems, vol. II. Springer, New York (2003)

20. Yao, YH, Postolache, M, Liou, YC: Strong convergence of a self-adaptive method for the split feasibility problem. Fixed Point Theory Appl. 2013, 201 (2013)

21. Yao, YH, Yang, PX, Kang, SM: Composite projection algorithms for the split feasibility problem. Math. Comput. Model. 57, 693-700 (2013)

22. Yao, YH, Liou, YC, Shahzad, N: A strongly convergent method for the split feasibility problem. Abstr. Appl. Anal. 2012, Article ID 125046 (2012)

23. Mohammad, E, Abdul, L: General split feasibility problems in Hilbert spaces. Abstr. Appl. Anal. 2013, Article ID 805104 (2013)

24. Censor, Y, Elfving, T: A multiprojection algorithm using Bregman projections in a product space. Numer. Algorithms 8 221-239 (1994)

25. Byrne, C: Iterative oblique projection onto convex sets and the split feasibility problem. Inverse Probl. 18, 441-453 (2002)

26. Wang, PY, Zhou, HY: A preconditioning method of the CQ algorithm for solving the extended split feasibility problem. J. Inequal. Appl. 2014, 163 (2014). doi:10.1186/1029-242X-2014-163

27. Yang, Q: On variable-step relaxed projection algorithm for variational inequalities. J. Math. Anal. Appl. 302, 166-179 (2005)

28. López, G, Martín-Márquez, M, Wang, F, Xu, H-K: Solving the split feasibility problem without prior knowledge of matrix norms. Inverse Probl. 28, 085004 (2012)

29. Wang, Z, Yang, Q, Yang, Y: The relaxed inexact projection methods for the split feasibility problem. Appl. Math Comput. 217, 5347-5359 (2011)

30. Yang, Q: The relaxed CQ algorithm solving the split feasibility problem. Inverse Probl. 20, 1261-1266 (2004)

10.1186/1029-242X-2014-435

Cite this article as: Wang et al.: Preconditioning methods for solving a general split feasibility problem. Journal of Inequalities and Applications 2014, 2014:435

\section{Submit your manuscript to a SpringerOpen ${ }^{\circ}$ journal and benefit from:}

- Convenient online submission

Rigorous peer review

- Immediate publication on acceptance

- Open access: articles freely available online

- High visibility within the field

- Retaining the copyright to your article 